\title{
COMERCIO INTRAINDUSTRIAL Y EQUILIBRIO INTEGRADOR: MODELO HECKSHER-OHLIN
}

\author{
INTRAINDUSTRIAL TRADE AND INTEGRATED BALANCE: \\ HECKSHER-OHLIN MODEL
}

Francisco Andrade Domínguez

Docente, Universidad Laica Vicente Rocafuerte de Guayaquil ULVR, (Ecuador). E-mail: fandraded@ulvr.edu.ec ORCID: https://orcid.org/0000-0003-2948-9965

Mónica Villamar Mendoza

Docente, Universidad Laica Vicente Rocafuerte de Guayaquil ULVR, (Ecuador).

E-mail: mvillamarm@ulvr.edu.ec ORCID: https://orcid.org/0000-0002-2091-7153

Betty Aguilar Echeverría

Docente, Universidad Laica Vicente Rocafuerte de Guayaquil ULVR, (Ecuador). E-mail: baguilare@ulvr.edu.ec ORCID: https://orcid.org/0000-0001-7507-0212

\section{Evangelina Méndez Encalada}

Docente, Universidad Laica Vicente Rocafuerte de Guayaquil ULVR, (Ecuador).

E-mail: emendeze@ulvr.edu.ec ORCID: https://orcid.org/0000-0002-0678-9933

Recepción: 07/11/2019 Aceptación: 16/01/2020 Publicación: 25/05/2020

\section{Citación sugerida:}

Andrade, F., Villamar, M., Aguilar, B., y Méndez, E. (2020). Comercio intraindustrial y equilibrio integrador: Modelo Hecksher-Ohlin. 3C Empresa. Investigación y pensamiento crítico, 9(2), 17-39. https://doi. org/10.17993/3cemp.2020.090242.17-39 


\section{RESUMEN}

Los antecedentes del estudio se relacionan con la economía internacional, la reducción de costos de transporte, la facilidad de las transacciones informáticas, la constitución de corresponsalías, y la apertura del periodo de paz más largo de los últimos tiempos, constituyen un espacio para la internacionalización de los negocios.

El objetivo de este artículo es analizar las implicaciones que tiene el comercio Intraindustrial y equilibrio integrador basados en el modelo Hecksher-Ohlin (HO), para determinar posibles escenarios que enfrentan los países en el comercio Intraindustrial, así como la movilidad de los factores de producción como limitación del modelo y opciones de libre movilidad de bienes entre 2 países.

Los principales resultados se obtuvo mediante la aplicación de una metodología analítica basada en el estudio de las principales variables de tipo transversal, se desarrolló ecuaciones factoriales donde se obtuvo varios resultados, uno de ellos es la ventaja que el país local obtiene cuando es intensivo capital, lo cual da como resultado que $\mathrm{r}-\mathrm{w}=(1,62)$, porque los trabajadores aprovechan su escasez relativa; mientras que $*_{r-w} * *$ país extranjero gana un trabajador $(1,22)$, esto trae como principales resultado que los dos países sean beneficiados por el comercio ya que el equilibrio mundial se da con especialización total.

Como conclusión se establecen, que los países con factor intensivo en conocimiento son quienes decidan si tiene libre de movilidad de factores.

\section{PALABRAS CLAVE}

Comercio, Intraindustrial, Economía Internacional, Equilibrio integrador. 


\section{ABSTRACT}

The background of the study is related to the international economy, the reduction of transport costs, the ease of computer transactions, the establishment of correspondents, and the opening of the longest peace period of recent times, constitute a space for internationalization of bussiness.

The objective of this article is to analyze the implications of Intra-industrial trade and integrative balance based on the Hecksher-Ohlin (HO) model, to determine possible scenarios that countries face in Intra-industrial trade, as well as the mobility of the factors of production like limitation of the model and options of free mobility of goods between 2 countries. The main results were obtained through the application of an analytical methodology based on the study of the main crosssectional variables, factorial equations were developed where several results were obtained, one of them is the advantage that the local country obtains when it is capital intensive, which results in rw =(1.62), because workers take advantage of their relative scarcity; While * $r$-w ** foreign country earns a worker (1.22), this brings as a main result that the two countries are benefited by trade since the global balance is given with full specialization.

In conclusion, it is established that countries with knowledge-intensive factors are the ones who decide if they have factor-free mobility.

\section{KEYWORDS}

Trade, Intraindustrial, International Economy, Integrative Balance. 


\section{INTRODUCCIÓN}

Partimos del análisis del modelo de comercio internacional de Hecksher-Ohlin (HO) pilar de la economía internacional estándar. Sus limitaciones se han discutido e implementado mediante una variedad de contribuciones que han podido incluir en su marco los efectos del comercio en los mercados internos, el análisis de la movilidad de los factores de producción, la variedad de gustos y preferencias entre países. En ese sentido los límites de los supuestos básicos sobre los determinantes de la especialización de los países comerciantes son aspectos que aún no se han abordado de manera puntual.

El modelo HO se basa en el supuesto de que la especialización de los países comerciales es exógena; es evidente entonces que la versión simple del modelo HO no puede explicar varios hechos estilizados que han caracterizado la evolución del comercio internacional en las últimas décadas.

Desde la temprana contribución de Leontief (1953), la evidencia sugiere que los países ricos en capital se especializan y exportan productos abundantes en capital físico (Highly Skilled Labor, Unskilled Labor) e importan capital intensivo (Rubiano-Matulevich, 2013).

Hecha la observación anterior el modelo de HO no puede acomodar fácilmente el papel del conocimiento como un aporte calificado y sus efectos en la división internacional del trabajo. También es difícil conciliar el impresionante crecimiento de la industria intraindustrial mientras el comercio con el modelo $\mathrm{HO}$ enfatiza el comercio interindustrial.

Este argumento permite tener mercados más grandes, acceder a materias primas de las que no disponemos, conseguir financiamiento cuando es un buen momento para invertir localmente y encontrar opciones diversificadas para ahorrar en el extranjero, en ese mismo sentido David Hume desarrolló su teoría para demostrar que no se podía mantener a la economía exportando grandes cantidades e importando casi nada todo el tiempo (Urbano, 2000). 
Los hechos estilizados de la evolución de los costos de los factores tampoco se ajustan al modelo HO simple. La evidencia muestra la resistencia de los salarios nominales y reales en los países avanzados, junto con el rápido aumento de los costos laborales en los recién llegados, tanto como los costos de los usuarios de capital exhiben una disminución secular que no coincide con las expectativas basadas en el modelo HO sobre su aumento acelerado después de la entrada de mano de obra abundante o recién llegados. El modelo $\mathrm{HO}$ no proporciona herramientas para comprender los determinantes del cambio estructural que sigue a la integración en los mercados internacionales de productos.

Finalmente, y lo más importante, el modelo HO supone que un país con desventaja va a recibir más contenido factorial de factores exógenos. La hipótesis de que un estado en equilibrio integrador supone introducción de libre comercio debido a la evolución del comercio internacional, la entrada de nuevos competidores y los cambios consiguientes en los mercados de productos y factores no se tiene en cuenta tanto como la hipótesis, respaldada por mucha evidencia empírica, de que la especialización de los países e incluso sus costos de factores relativos pueden determinarse de manera endógena.

Es evidente entonces que el análisis de la globalización de los mercados de productos y factores que ha tenido lugar desde fines del siglo XX proporciona una gran evidencia sobre el papel crucial del cambio tecnológico y las leyes de acumulación del conocimiento tecnológico que permite su introducción que necesita integrarse en el marco básico del modelo de HO (Montobbio y Rampa, 2005; Romer, 2013; Baldwin, 2016).

Después de las consideraciones anteriores Schumpeter (1947) da una respuesta creativa y elaborada por la integración de las naciones y de congruencia tecnológica (Antonelli, 2017) concluyen que la especialización de los socios comerciales, en cada momento, son un resultado de un proceso endógeno de cambio tecnológico provocado por la respuesta creativa a los desafios planteados por la integración en los mercados internacionales de nuevos socios comerciales y basada en la generación de conocimiento tecnológico como una entrada endógena hace posible superar estas limitaciones. 
Hechas las consideraciones anteriores el resto del documento está organizado de la siguiente manera. La sección 2 resume los fundamentos de la respuesta creativa schumpeteriana. La Sección 3 elabora su aplicación para implementar un enfoque schumpeteriano de la $\mathrm{HO}$ que elabora un modelo (SHO) de comercio internacional con tres versiones que muestran cómo las condiciones fuera de equilibrio de los mercados de productos y factores desencadenadas por la entrada de nuevos (grandes) países con mano de obra abundante.

\section{REVISIÓN DE LA LITERATURA}

La integración del legado schumpeteriano en el modelo HO permite articular un marco de trabajo Schumpeter-Hecksher-Ohlin (SHO) que puede acomodar el análisis de la dinámica de la acumulación de conocimiento en libre mercado.

El injerto de la respuesta creativa schumpeteriana en la economía del comercio internacional permite articular un marco que muestra cómo las condiciones cambiantes de los mercados de productos y factores tienen el doble efecto de agitar las tasas de cambio tecnológico y sesgar su dirección: rara vez es neutral el cambio tecnológico (Blanchard, 1997; Moronta y Gisbert, 2017; Impullitti y Licandro, 2017).

El marco del modelo SHO proporciona las herramientas para comprender la dinámica de la especialización de los países, la evolución de los costos de los factores relativos y los cambios estructurales de los países avanzados, formados por el rápido declive de las industrias manufactureras y su progreso discontinuo.

La sustitución con las nuevas industrias de servicios de negocios intensivos en conocimiento (Highly Skilled Labor), da como resultado endógeno la respuesta creativa de las empresas que intentan hacer frente a las condiciones cambiantes de los mercados internacionales de productos y factores traídos por la globalización (Pérez, 1996). 
Los cambios radicales en la especialización de los países tanto avanzados como industrializados que han tenido lugar desde las últimas décadas del siglo XX pueden considerarse como la consecuencia de la radicalidad; cambios tecnológicos y estructurales introducidos para hacer frente a la globalización de los mercados de productos y factores (Freeman, 1996; Pérez, 2002).

El núcleo de la teoría económica de Schumpeter (1947) elaboró la noción de innovación como una respuesta creativa provocada por un desajuste entre las condiciones esperadas y reales de los mercados de productos y factores (Sung, 2019). Las posibles respuestas de las empresas pueden ser adaptativas o creativas. Precisando de una vez la respuesta puede ser simplemente adaptativa cuando consiste solo en ajustes técnicos tradicionales de precio / cantidad (en oposición a los tecnológicos).

La respuesta es adaptativa cuando las empresas no pueden generar la cantidad adecuada de nuevo capital intensivo y no pueden innovar, la respuesta creativa de las empresas es, de hecho, solo posible cuando y donde las externalidades de conocimiento específicas hacen posible la generación recombinante de nuevo conocimiento tecnológico a costos que están por debajo del nivel de equilibrio (Viguera, Alpizar, Harvey, Martínez-Rodríguez, y Saborío-Rodríguez, 2019). En efecto, tales externalidades relevantes del conocimiento material están disponibles en los sistemas económicos donde la generación de conocimiento tecnológico es el resultado de la participación activa y la interacción de una miríada de innovadores que pueden acceder al acervo de conocimiento casi público integrado en el entorno institucional y acumulado a través del tiempo (Antonelli, 2017).

Los recientes avances de la economía del conocimiento que consideran el conocimiento tecnológico como una actividad colectiva y recombinante con fuertes características sistémicas contribuyen (Pérez, 2015).

La implementación del enfoque schumpeteriano basa su análisis en la apreciabilidad y exhaustividad limitadas del conocimiento y explora sus consecuencias sobre la generación y explotación del conocimiento. En esta literatura, los derrames de conocimientos y, en general, el conocimiento externo 
de cada empresa es indispensable para la generación de nuevos conocimientos. Debido al contenido pegajoso y tácito del conocimiento, se necesitan esfuerzos dedicados e intencionales e interacciones localizadas entre productores y usuarios para utilizar el conocimiento externo en la generación de nuevo conocimiento. Las externalidades del conocimiento son pecuniarias más que puras (Pérez, 2015).

Significa entonces que la organización del sistema en términos de condiciones de acceso al conjunto externo de conocimiento tecnológico es el ingrediente crucial y complementario, junto con la calidad e intensidad de los esfuerzos de investigación interna, que hace posible la introducción endógena de innovaciones (Antonelli, 2017).

Los agentes tienen éxito en su respuesta creativa cuando se aplican una serie de condiciones externas contingentes a nivel del sistema (Antonelli y Colombelli, 2011).

La innovación es el resultado de la acción económica colectiva de los agentes: la innovación es un proceso colectivo dependiente de la ruta que tiene lugar en un contexto localizado, si, cuándo y dónde el costo de usar y acceder al stock de conocimiento cuasi público permite generar nuevos conocimientos a costos que son por debajo de los niveles de equilibrio y un número suficiente de respuestas creativas se realizan de manera coherente, complementaria y consistente.

Cabe agregar, que la innovación es una de las propiedades emergentes clave de un sistema económico que tiene lugar cuando la complejidad está 'organizada', es decir, cuando una serie de condiciones complementarias permiten la reacción creativa de los agentes y permiten introducir innovaciones que realmente aumentan su eficiencia (Cordero Espinosa y Arias Figueroa, 2018).

La cantidad de externalidades e interacciones de conocimiento disponibles para la empresa integrada en el sistema influye en su capacidad para generar nuevo conocimiento tecnológico y, en consecuencia, la posibilidad real de hacer que su reacción sea creativa en lugar de adaptativa e introducir cambios tecnológicos reales (Antonelli, 2017). 
Debido al énfasis schumpeteriano en los desajustes entre el factor esperado y el real, y no solo las condiciones del mercado del producto, el marco de cambio tecnológico localizado acomoda el núcleo analítico de la literatura de cambio tecnológico inducido. Esta literatura reconoce que la tasa y la dirección del cambio tecnológico son inducidas por las condiciones cambiantes de los mercados de factores (Ruttan, 2001). Cuanto más grandes son los cambios en los mercados de factores mayores son las tasas de introducción factorial en innovación (Antolín-López, Martínez-del-Río, y Céspedes-Lorente, 2016).

El cambio tecnológico está intrínsecamente sesgado, es decir, es intensivo en capital y, por lo tanto, ahorro de mano de obra, o intensivo en mano de obra y, por lo tanto, ahorro de capital. el resultado del intento de los innovadores de aprovechar las oportunidades y limitaciones de los mercados de factores.

La abundancia relativa de un factor de producción, como el trabajo calificado o el conocimiento tecnológico, induce la introducción de innovaciones sesgadas hacia un uso intensivo de habilidades o conocimiento.

La noción de congruencia tecnológica juega un papel central en el enfoque de cambio tecnológico localizado; la congruencia tecnológica consiste en la correspondencia entre los insumos localmente abundantes y su elasticidad de salida.

Cabe agregar que la congruencia tecnológica es alta cuando la elasticidad de salida de un insumo, digamos conocimiento, es grande en un país donde el conocimiento es abundante. Los altos niveles de congruencia tecnológica conducen a altos niveles de productividad total de los factores.

El aumento en los niveles de congruencia tecnológica desencadena efectos de sesgo positivo, que, junto a los cambios, permite aumentar los niveles de productividad total del factor (Naimzada y Tramontana, 2015; 29). 
En este propósito podemos plantear varios resultados importantes:

- Teorema schumpeteriano es congruente con la innovación como una respuesta creativa provocada por un desajuste entre las condiciones esperadas y reales de los mercados de productos y factores.

- Modelo Hecksher-Ohlin, las exportaciones del país relativamente abundante en capital serán del bien que utiliza de forma relativamente intensa en este factor. El país relativamente escaso de capital importará este bien y exportará a cambio el que usa de forma relativamente intensa mano de obra.

- Teorema de Igualación de Precios conjuga que el libre comercio de bienes generará convergencia en el precio de los factores de producción que se usan en los dos países. De esta manera, la libre movilidad de bienes es un sustituto de la libre movilidad de factores de producción.

- Teorema de Stolper-Samuelson liga al cambio en los precios de los factores entre el cambio en los ingresos de los dueños de estos. Los dueños del factor relativamente abundante en un país verán crecer sus ingresos con el libre comercio de bienes, mientras que los dueños de los factores relativamente escasos reducirán sus ingresos.

\section{METODOLOGÍA}

En esta investigación se realiza se desarrolla el modelo económico de Hecksher y Ohlin desarrollaron una modelación con 2 países en el que analizan esta situación.

En la versión más sencilla hay 2 países, 2 factores de producción (capital físico y mano de obra) y 2 bienes.

- La tecnología de producción es la misma para cada país en cada bien.

- Las dotaciones de factores son diferentes.

- Hay libre movilidad de factores entre industrias de un mismo país. 
- No hay movilidad de factores entre países.

La producción supondremos que es del tipo Cobb-Douglas (Sánchez, 2011):

$$
Y_{1}=A k_{1}^{\alpha} L_{1}^{1-\alpha} Y_{2}=B k_{2}^{\beta} L_{2}^{1-\beta}
$$

No es necesaria esta estructura, pero nos simplificará los cálculos.

Supondremos que $\alpha>\beta$ para que la industria del bien 1 sea relativamente intensa en capital y la del bien 2 relativamente intensa en mano de obra.

La intensidad relativa significa que todos los precios de factores, la industria intensa relativamente usa ese factor más proporcionalmente.

$$
\forall r, w, \frac{k_{1}}{L_{1}}>\frac{k_{2}}{L_{2}}
$$

De igual manera las dotaciones de factores de cada país (que se representan con la notación habitual del asterisco para las variables del país extranjero) son: $\bar{K}$ y $\bar{L}$ para el país local $\bar{K}^{*}$ y $\bar{L}^{*}$. Las dotaciones no serán en general iguales y podemos definir la abundancia relativa de cada país de la siguiente manera:

Suponiendo $\frac{\bar{K}}{\bar{L}}>\frac{\bar{K}^{*}}{\bar{L}^{*}}$, entonces diríamos que le país local es relativamente abundante en capital. Note que podría tener menos capital y aun así ser relativamente abundante en capital. En este caso la dotación de factores genera el efecto de la ventaja comparativa.

Las empresas deben encontrar la mejor combinación de factores para producir dado los precios de estos:

$$
\max P_{1} A K_{1}^{\alpha} L_{1}^{1-\alpha}-w L_{1}-r K_{1}
$$

Esto nos da las condiciones de primer orden:

$$
r=P_{1} \alpha A\left(\frac{L_{1}}{K_{1}}\right)^{1-\alpha} w=P_{1}(1-\alpha)\left(\frac{K_{1}}{L_{1}}\right)^{\alpha}
$$


Obviamente tendremos unas ecuaciones similares para la industria que produce el bien. Adicionalmente tenemos las condiciones de vaciado de mercado de factores:

$$
K_{1}+K_{2}=\bar{K} \text { y } L_{1}+L_{2}=\bar{L}
$$

Y como mercados competitivos con tecnologías de retornos constantes a escala no generan ganancia:

$$
P_{1} Y_{1}=w L_{1}+r K_{1} P_{2} Y_{2}=w L_{2}+r K_{2}
$$

Por simplicidad suponemos que la utilidad también es del tipo Cobb-Douglas con parámetro 0.5. De modo que el gasto en ambos productos será igual. Para el caso de autarquía donde cada país produce y consume esa misma cantidad de cada bien, tenemos:

$$
P_{1} Y_{1}=P_{2} Y_{2}
$$

Con estas ecuaciones podemos encontrar el equilibrio en autarquía:

$$
\begin{gathered}
K_{1}=\frac{\alpha}{\alpha+\beta} \bar{K}, \quad K_{2}=\frac{\beta}{\alpha+\beta} \bar{K} \\
L_{1}=\frac{1-\alpha}{2-\alpha-\beta} \bar{L}, \quad L_{2}=\frac{1-\beta}{2-\alpha-\beta} \bar{L} \\
C_{1}=Y_{1}=A K_{1}^{\alpha} L_{1}^{1-\alpha}, \quad C_{2}=Y_{2}=B K_{2}^{\beta} L_{2}^{1-\beta} \\
P_{1}=\frac{C_{2}}{C_{1}}, \quad P_{2}=1 \\
w=(1-\beta) B\left(\frac{K_{2}}{L_{2}}\right)^{\beta}, r=\beta B\left(\frac{L_{2}}{K_{2}}\right)^{1-\beta}
\end{gathered}
$$

Para el caso de comercio internacional, las ecuaciones cambiarían porque ya las cantidades consumidas y producidas de cada bien, en cada país, no tienen por qué ser iguales.

Los países se especializarán (no totalmente) en la producción del bien que use más del factor abundante en ese país (todo en términos relativos). Pero el consumo de los dos bienes tenderá a ser más bien combinado en una canasta. 
Lo que si debe ocurrir es que los precios de los bienes sean iguales en los dos países ya que si no muchos agentes querrán comprar en el país donde es barato para vender en el país caro. Este proceso haría subir el precio en el país barato por el exceso de demanda y lo haría bajar en el país caro por el exceso de oferta.

Esto ocurrirá hasta que los precios se igualen.

Las ecuaciones de autarquía:

$$
P_{1} Y_{1}=P_{2} Y_{2} P_{1}^{*} Y_{1}^{*}=P_{2}^{*} Y_{2}^{*} P_{2}=P_{2}^{*}=1
$$

Cambiaran por las siguientes:

$$
\begin{gathered}
P_{1} Y_{1}+P_{1}^{*} Y_{1}^{*}=P_{2} Y_{2}+P_{2}^{*} Y_{2}^{*} P_{1}=P_{1}^{*} \\
P_{2}=P_{2}^{*}=1
\end{gathered}
$$

Las demás ecuaciones se mantienen. Por eso el resultado se obtiene de las ecuaciones:

$$
\begin{gathered}
K_{1}^{M}=\frac{\alpha}{\alpha+\beta}\left(\bar{K}+\bar{K}^{*}\right), \quad K_{2}^{M}=\frac{\beta}{\alpha+\beta}\left(\bar{K}+\bar{K}^{*}\right) \\
L_{1}^{M}=\frac{1-\alpha}{2-\alpha-\beta}\left(\bar{L}+\bar{L}^{*}\right), \quad L_{2}^{M}=\frac{1-\beta}{2-\alpha-\beta}\left(\bar{L}+\bar{L}^{*}\right) \\
C_{1}^{M}=Y_{1}^{*}=A\left(K_{1}^{M}\right)^{\alpha}\left(L_{1}^{M}\right)^{1-\alpha}, \quad C_{2}^{M}=Y_{2}^{M}=B\left(K_{2}^{M}\right)^{\beta}\left(L_{2}^{M}\right)^{1-\beta} \\
P_{1}^{M}=\frac{C_{2}^{M}}{C_{1}^{M}}, P_{2}^{M}=1 \\
w^{M}=(1-\beta) B\left(\frac{K_{2}^{M}}{L_{2}^{M}}\right)^{\beta}, r^{M}=\beta B\left(\frac{L_{2}^{M}}{K_{2}^{M}}\right)^{1-\beta}
\end{gathered}
$$

Las posibles respuestas pueden ser adaptativas o creativas. La respuesta puede ser simplemente adaptativa cuando consiste solo en ajustes técnicos tradicionales de precio / cantidad (en oposición a los tecnológicos). La respuesta es adaptativa cuando no pueden generar la cantidad adecuada de nuevos conocimientos tecnológicos y no pueden innovar (Vidal, 2010). 


\section{RESULTADOS}

Tabla 1. Parámetros de los Países.

\begin{tabular}{|c|c|c|c|}
\hline \multicolumn{4}{|c|}{ Parámetros } \\
\hline A & & 1 & \\
\hline alfa & & 0,4 & \\
\hline B & & 1 & \\
\hline beta & & 0,5 & \\
\hline \multicolumn{4}{|c|}{ FACTORES } \\
\hline $\mathrm{K}$ barra & 4 & $\mathrm{~K}$ barra* & 5 \\
\hline L barra & 3 & L barra* & 5 \\
\hline
\end{tabular}

Fuente: elaboración propia.

$$
\bar{K}=4, \quad \bar{L}=3, \bar{K}^{*}=5, \bar{L}^{*}=5
$$

Tabla 2. Países en Autarquía.

\begin{tabular}{|c|c|c|c|}
\hline \multicolumn{2}{|c|}{ País local } & \multicolumn{2}{c|}{ País extranjero } \\
\hline K1 & 1,777777778 & K1 $^{*}$ & 2,22222222 \\
\hline K2 & 2,222222222 & K2 $^{*}$ & 2,77777778 \\
\hline L1 & 1,636363636 & L1 $^{*}$ & 2,72727273 \\
\hline L2 & 1,363636364 & L2 $^{*}$ & 2,27272727 \\
\hline C1 & 1,691526793 & C1 $^{*}$ & 2,51276652 \\
\hline C2 & 1,74077656 & C2 $^{*}$ & 2,51259454 \\
\hline Y1 & 1,691526793 & Y1 $^{*}$ & 2,51276652 \\
\hline Y2 & 1,74077656 & Y2 $^{*}$ & 2,51259454 \\
\hline r & 0,391674726 & $\mathrm{r}^{*}$ & 0,45226702 \\
\hline w & 0,638284739 & $\mathrm{~W}^{*}$ & 0,5527708 \\
\hline P1 & 1,02911557 & P1 & 0,99993156 \\
\hline P2 & & P2 & \\
\hline & & & 1 \\
\hline
\end{tabular}

Fuente: elaboración propia.

Análisis:

* El país local (Todo lo que produce consume en "autarquía” C2 Y2) aprovecha su escasez relativa. 
* Ligeramente hay una mayor producción en el B1 que el b2 en el país local, porque el bien 2 es intensivo.

* r-w país local el trabajador gana 1,62, por los trabajadores aprovechan su escasez relativa.

* r-w **país extranjero gana un trabajador 1,22.

Tabla 3. Parámetros de los Países.

Fuente: elaboración propia.

\begin{tabular}{|c|c|}
\hline \multicolumn{2}{|c|}{ Parámetros } \\
\hline A & 1 \\
\hline alfa & 0,4 \\
\hline B & 1 \\
\hline beta & 0,5 \\
\hline K barra & 12 \\
\hline L barra & 12 \\
\hline
\end{tabular}

Tabla 4. Países en Equilibrio Integrador.

Fuente: elaboración propia.

\begin{tabular}{|c|c|}
\hline \multicolumn{2}{|c|}{ Equilibrio integrado } \\
\hline K1 & 5,333333333 \\
\hline K2 & 6,666666667 \\
\hline L1 & 6,545454545 \\
\hline L2 & 5,454545455 \\
\hline C1 & 6,030639639 \\
\hline C2 & 6,030226892 \\
\hline Y1 & 6,030639639 \\
\hline Y2 & 6,030226892 \\
\hline r & 0,452267017 \\
\hline w & 0,552770798 \\
\hline P1 & 0,999931558 \\
\hline P2 & 1 \\
\hline
\end{tabular}


Análisis:

* El libre comercio de bienes generará convergencia en el precio de los factores de producción que se usan en los dos países. La libre movilidad de bienes es un sustituto de la libre movilidad de factores de producción.

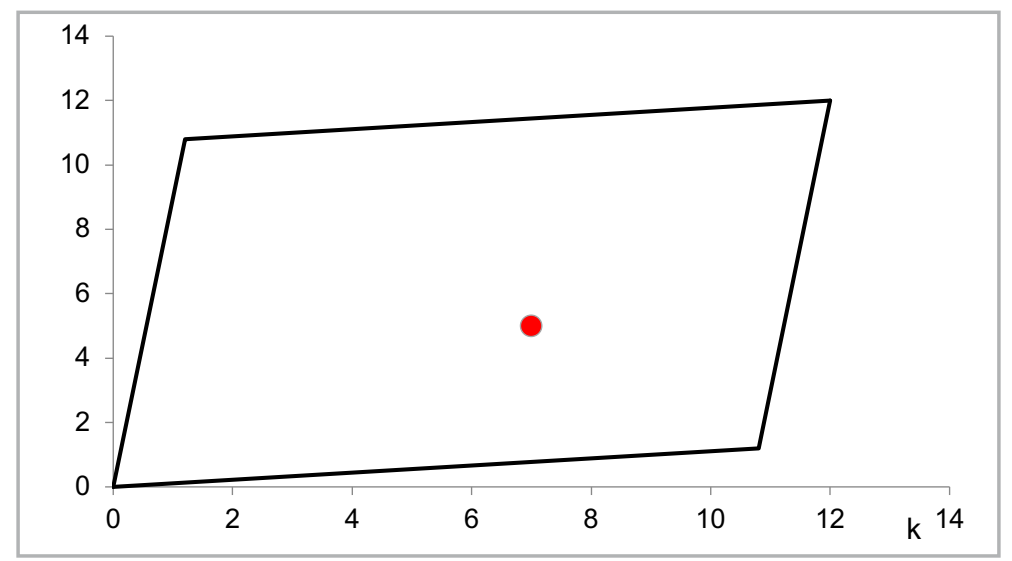

Figura 1. Equilibrio Integrador. Fuente: elaboración propia.

Análisis:

* Se explica que siempre que el punto rojo está adentro del paralelo el comercio exterior es un sustituto de la libre movilidad factores.

Tabla 5. Parámetros de los Países.

Fuente: elaboración propia.

\begin{tabular}{|c|c|c|c|}
\hline \multicolumn{4}{|c|}{ PARÁMETROS } \\
\hline$A$ & & \multicolumn{2}{|c|}{1} \\
\hline alfa & & \multicolumn{2}{|c|}{0,9} \\
\hline B & & \multicolumn{2}{|c|}{1} \\
\hline beta & & \multicolumn{2}{|c|}{0,1} \\
\hline \multicolumn{4}{|c|}{ FACTORES } \\
\hline $\mathrm{K}$ barra & 7 & K barra* & 7 \\
\hline L barra & 5 & L barra* & 5 \\
\hline
\end{tabular}




$$
\bar{K}=4, \quad \bar{L}=3, \bar{K}^{*}=5, \bar{L}^{*}=5
$$

Tabla 6. Países en Libre Comercio

\begin{tabular}{|c|c|c|c|}
\hline \multicolumn{2}{|c|}{ País Local } & \multicolumn{2}{|c|}{ País extranjero } \\
\hline K1 & 6,525 & $\mathrm{~K} 1^{*}$ & 6,525 \\
\hline K2 & 0,475 & $\mathrm{~K}^{*}{ }^{*}$ & 0,475 \\
\hline L1 & 0,725 & $\mathrm{~L} 1^{*}$ & 0,725 \\
\hline L2 & 4,275 & $\mathrm{~L}^{*}$ & 4,275 \\
\hline C1 & 4,334804434 & $\mathrm{C} 1^{*}$ & 4,334804434 \\
\hline $\mathrm{C} 2$ & 4,334804434 & $\mathrm{C} 2 *$ & 4,334804434 \\
\hline Y1 & 5,23788869 & $\mathrm{Y} 1^{*}$ & 5,23788869 \\
\hline Y2 & 3,431720177 & $Y 2^{*}$ & 3,431720177 \\
\hline$r$ & 0,722467406 & $r^{*}$ & 0,722467406 \\
\hline$w$ & 0,722467406 & $w^{*}$ & 0,722467406 \\
\hline P1 & 1 & $\mathrm{P} 1^{*}$ & 1 \\
\hline P2 & 1 & $\mathrm{P} 2^{*}$ & 1 \\
\hline
\end{tabular}

Fuente: elaboración propia.

Análisis:

* r-w país local pierde sus escases relativa.

* r-w **país extranjero gana un trabajador 1.

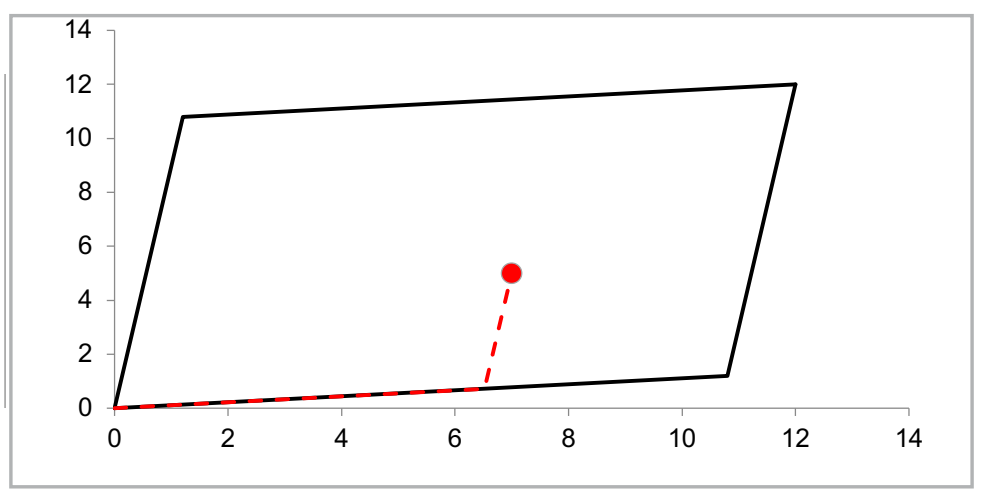

Figura 2. Equilibrio Integrador. Fuente: elaboración propia. 
Análisis:

* La línea roja me está diciendo lo que el país local produce del b1 en el mundo esta parte vertical; lo hace el país local.

* La línea roja ascendente me está diciendo que el país local de toda la producción del b2 en el mundo esta parte lo hace el país local.

* Siempre que el punto rojo está adentro del paralelo el comercio exterior es un sustituto de la libre movilidad factores.

\subsection{PATRONES DEL COMERCIO}

Tabla 7. Parámetros de los Países.

\begin{tabular}{|c|c|c|c|}
\hline \multicolumn{4}{|c|}{ PARÁMETROS } \\
\hline \multicolumn{2}{|l|}{$A$} & \multicolumn{2}{|l|}{1} \\
\hline \multicolumn{2}{|l|}{ alfa } & \multicolumn{2}{|l|}{0,9} \\
\hline \multicolumn{2}{|l|}{$B$} & \multicolumn{2}{|l|}{1} \\
\hline \multicolumn{2}{|l|}{ beta } & \multicolumn{2}{|l|}{0,1} \\
\hline \multicolumn{4}{|c|}{ FACTORES } \\
\hline $\mathrm{K}$ barra & 4 & $\mathrm{~K}$ barra* & 5 \\
\hline L barra & 3 & L barra* & 5 \\
\hline
\end{tabular}

Fuente: elaboración propia.

$$
\bar{K}=4, \quad \bar{L}=3, \bar{K}^{*}=5, \bar{L}^{*}=5
$$

Tabla 8. Comparación de resultados.

Fuente: elaboración propia.

\begin{tabular}{|c|c|c|}
\cline { 2 - 3 } \multicolumn{1}{c|}{} & C1 & C2 \\
\hline \multirow{2}{*}{ Autarquía } & 4,8899396 & 3,7359501 \\
\hline Comercio & 5,23788869 & 3,43172018 \\
\hline Autarquía* & 4,33480443 & 4,33480443 \\
\hline Comercio* & 4,8899396 & 3,7359501 \\
\hline & 5,23788869 & 3,43172018 \\
\hline
\end{tabular}


Análisis:

*El libre comercio de bienes generará convergencia en el precio de los factores de producción que se usan en los dos países. De esta manera, la libre movilidad de los factores de producción es posible.

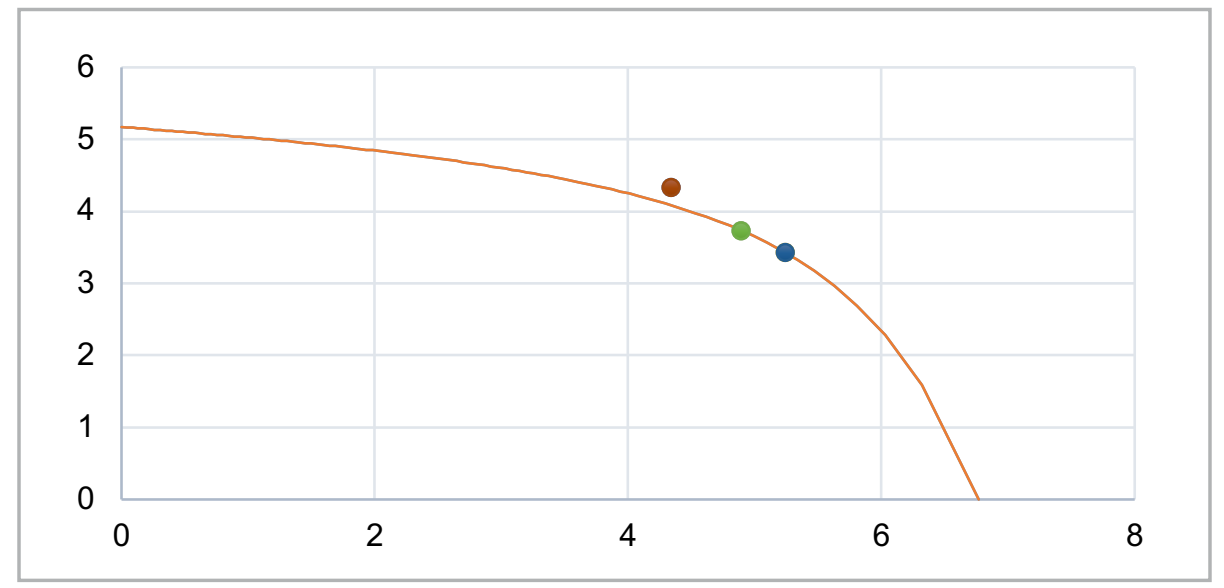

Figura 3. Países en Bienestar. Fuente: elaboración propia.

\section{CONCLUSIONES}

El comercio Intraindustrial y equilibrio integrador basados en el modelo Hecksher-Ohlin se dan por la igualdad en el comercio internacional, así como el principio de la no discriminación. El objetivo de la investigación se centra en la aplicación de escenarios para los países en vías de desarrollo, identificando a la discriminación de precios como un principio no valido en los escenarios de producción; ya que ellos necesitan preferencias para poder desarrollarse.

El propósito del análisis económico sobre el modelo HO, es hacer una combinación de convergencia en el precio de los factores de producción, utilizando ideas duopolistas se determina que la opción de elegir uno u otro, cantidad de oferta o precio, como parámetro de acción; en el comercio intraindustrial 
y equilibrio integrador, en conclusión, el principal resultado en la investigación demuestra que solo los países con factor intensivo en conocimiento son quienes decidan si tiene libre de movilidad de factores.

Por otra parte, la libre movilidad de bienes vs la libre movilidad de factores de producción demuestra que existen diferentes situaciones entre los países con sus ventajas definidas. Esto se trata bajo el título de libre movilidad factores, como se aplicó bajo los puestos teóricos en los tres escenarios distintos; el equilibrio integrador, libre mercado y bienestar, uno para cada escenario entonces la lógica puede ser rectificada.

\section{REFERENCIAS BIBLIOGRÁFICAS}

Antolín-López, R., Martínez-del-Río, J., y Céspedes-Lorente, J. (2016). Fomentando la innovación de producto en las empresas nuevas:¿ ¿ué instrumentos públicos son más efectivos? European Research on Management and Business Economics, 22(1), 38-46. https://doi.org/10.1016/j. iedee.2015.05.002

Antonelli, G. (2017). Endogenous Innovation. The Economics of an Emergent System Property. Cheltenham: Edward Elgar.

Antonelli, G., y Colombelli, A. (2011). Chapter 1 Globalization and Directed Technological Change at the Firm Level: The European Evidence. En Libecap, G. y Hoskinson, S. (Ed.) Entrepreneurship and Global Competitiveness in Regional Economies: Determinants and Policy Implications (Advances in the Study of Entrepreneurship, Innovation and Economic Growth, Vol. 22), Emerald Group Publishing Limited, Bingley, pp. 1-20. https://doi.org/10.1108/S1048-4736(2011)000002200

Baldwin, R. (2016). The Great Convergence Information Technology and the New Globalization. Harvard University Press.

Blanchard, O. J. (1997). The medium run. Brookings Papers on Economic Activity, 2, 89-141. https://www. brookings.edu/wp-content/uploads/2016/07/1997b_bpea_blanchard_nordhaus_phelps.pdf 
Cordero Espinosa, G. S., y Arias Figueroa, J. J. (2018). La innovación revelada por el margen extensivo en las exportaciones de las empresas proveedoras de servicios mineros en Chile. Revista TEC Empresarial, 12(2), 19-32. http://dx.doi.org/10.18845/te.v12i2.3718

Gristiano, A. (2019). The creative response and international trade. Structural Change and Economic Dynamics, 51, 445-452. https://doi.org/10.1016/j.strueco.2019.03.002

Freeman, C. (1996). International Library of Critical Writings in Economics. Edward Elgar Pub.

Impullitti, G., y Licandro, O. (2017). Trade, firm selection and innovation: the competition channel. The Economic Fournal, 128(608),189-229. https://doi.org/10.1111/ecoj.12466

Montobbio, F., y Rampa, F. (2005). The impact of technology and structural change on export performance in nine developing countries. World Development, Elsevier, 33(4), 527-547. https:// www.academia.edu/323114/The_Impact_of_Technology_and_Structural_Change_on_ Export_Performance_In_Nine_Developing_Countries

Moronta, I., y Gisbert, V. (2017). Entorno de competitividad en la República Dominicana. 3C Empresa, Investigacióny pensamiento crítico, (Edición especial), 64-72. http://dx.doi.org/10.17993/3cemp.2017. especial.64-72

Naimzada, A., y Tramontana, F. (2015). Dynamic properties of a Cournot-Bertrand duopoly game with differentiated products. Economic Modelling, 29(4), 1436-1439. https://doi.org/10.1016/j. econmod.2012.02.016

Pérez, A. (2015). ¿Por qué falla la economía? Economía Informa, 393, 82-98. https://doi.org/10.1016/j. ecin.2015.08.006 
Pérez, G. (1996). La modernización industrial en América Latina y la herencia de la sustitución de importaciones. Comercio exterior, 46(5), 347-363. http://www.carlotaperez.org/downloads/pubs/ ISILA.pdf

Pérez, G. (2002). Technological Revolutions and Financial Capital: The Dynamics of Bubbles and Golden Ages. Edward Elgar Publishing.

Romer, P. (2013). New goods, old theory, and the welfare costs of trade restrictions. Fournal of Development Economics, 43(1), 5-38. https://doi.org/10.1016/0304-3878(94)90021-3

Rubiano-Matulevich, E. (2013). El crecimiento de China e India y su relación con el patrón de especialización comercial de Estados Unidos, la Unión Europea y Colombia. Revista De La Maestría En Derecho Económico, 6(6), 87-142. https://revistas.javeriana.edu.co/index.php/revmaescom/ article/view/7169

Ruttan, V. (2001). Technology Growth and Development. An Induced Innovation Perspective. Oxford University Press.

Sánchez Álzate, M. (2011). Condicionan los recursos naturales el crecimiento económico? Semestre económico, 14(29), 117-128. https://doi.org/10.22395/seec.v14n29a6

Sung, N. S. (2019). Schumpeter on the Theory of Innovation and Competition: A Darwinian Interpretation. Trends and Outlook, 106, 196-228. https://www.kci.go.kr/kciportal/ci/ sereArticleSearch/ciSereArtiView.kci? sereArticleSearchBean.artiId=ART002470280

Urbano, F. (2000). Tolerancia y racionalidad: límites y presupuestos del análisis de la religión de David Hume. Revista de Filosofia, 34(1), 17-44. https://www.academia.edu/35885746/Tolerancia_y_ racionalidad_1\%C3\%ADmites_y_presupuestos_del_an $\%$ C 3\% A 1 lisis_de_la_religi $\%$ C $3 \%$ B 3 n de_David_Hume_Tolerance_and_Rationality_Limits_and_Presumption_in_David_Humes_ Analysis_of_Religion 
Vidal de la Rosa, G. (2010). Teoría democrática. Joseph Schumpeter y la síntesis moderna. Argumentos (México, DF), 23(62), 177-199. http://www.scielo.org:mx/scielo.php?script=sci_ abstract\&pid=S0187-57952010000100008\&lng=es\&nrm=iso

Viguera, B., Alpizar, F., Harvey, C., Martínez-Rodríguez, M., y Saborío-Rodríguez, M. (2019). Climate change perceptions and adaptive responses of small-scale coffee farmers in Costa Rica. Agronomía Mesoamericana, 30(2), 333-351. https://doi.org/10.15517/am.v30i2.32905 